\title{
"Evaluación ergonòmica del puesto de trabajo de un alimentador de grano en un proceso de molienda de sorgo"
}

\section{"Ergonomic evaluation of the workstation of a grain feeder in a sorghum milling process."}

\author{
DOI: $10.46932 / \mathrm{sfjdv2n2-049}$
}

Received in: january 1st, 2020

Accepted in: March 30th, 2020

\section{Omar García de la Rosa}

Dr. en Administración y Políticas Públicas.

Current Institution: Instituto Tecnológico de Toluca, Toluca México.

Full address: Av. Tecnológico S/N, col. Agrícola Bellavista, Metepec Edo. Méx., México. C.P. 52149

E-mail: omargarcia_1978@hotmail.com

\section{María de Lourdes Olivares Estrada}

Dr. en Administración y Políticas Públicas.

Current Institution: Centro de Estudios Tecnológicos Industrial y de Servicios No. 64, Toluca, México. Full address: Av. Benito Juárez Esq. Juan Escutia S/N, Col. San Mateo Oxtotitlán, Toluca Edo. Méx., México. C.P. 50100

E-mail: mloe09@yahoo.com.mx

\section{Angelica Ortega Soto \\ Maestra en Docencia.}

Current Institution: Centro de Estudios Tecnológicos Industrial y de Servicios No. 64, Toluca, México. Full address: Av. Benito Juárez Esq. Juan Escutia S/N, Col. San Mateo Oxtotitlán, Toluca Edo. Méx., México. C.P. 50100

E-mail: angiecetis64@gmail.com

\section{Guadalupe Ortega Rojas}

Ing. Agrónomo Fitotecnista.

Current Institution: Centro de Estudios Tecnológicos Industrial y de Servicios No. 64, Toluca, México. Full address: Av. Benito Juárez Esq. Juan Escutia S/N, Col. San Mateo Oxtotitlán, Toluca Edo. Méx., México. C.P. 50100

E-mail: Guadalupe_ortegarojas@yahoo.com.mx

\section{Ceciliano Hernández Leonardo}

Maestría en Ciencias (Física) .

Current Institution: Instituto Tecnológico de Toluca, Toluca México.

Full address: Av. Tecnológico S/N, col. Agrícola Bellavista, Metepec Edo. Méx., México. C.P. 52149

E-mail: lcecilianoh@yahoo.com.mx

\section{Rosenda García de la Rosa}

Maestría en Seguridad e Higiene Ocupacional.

Current Institution: Centro de Bachillerato Tecnológico del Gobierno del Estado de México

Full address: Carretera Amomoluco Santiago Tianguistengo Km. 7.5, Guadalupe Hidalgo, C.P.52756 Ocoyoacac, Méx

E-mail: garo_05@yahoo.com.mx 


\title{
Adiel García Vázquez
}

Maestría en Ciencias en Ingeniería Industrial.

Current Institution: Instituto Tecnológico de Toluca, Toluca México.

Full address: Av. Tecnológico S/N, col. Agrícola Bellavista, Metepec Edo. Méx., México. C.P. 52149

E-mail: adielgarciav@gmail.com

\section{Héctor García de la Rosa}

Dr. en Administración y Políticas Públicas.

Current Institution: Instituto Tecnológico de Toluca, Toluca México.

Full address: Av. Tecnológico S/N, col. Agrícola Bellavista, Metepec Edo. Méx., México. C.P. 52149

E-mail: hegar71@yahoo.com.mx

\section{RESUMEN}

En la investigación se presenta un estudio ergonómico relacionado con la necesidad de modificar las tareas que tradicionalmente se efectúan en la molienda de sorgo, tomando como base la conservación de la salud del jornalero en su puesto de trabajo, para proponer una adaptación de las condiciones laborales de operación, con la innovación de buenas prácticas en el proceso productivo. Inicialmente se realizó la descripción del proceso de producción de harina de sorgo, mismo que se utiliza en la formulación de alimento balanceado para ganado bovino y posteriormente se realizó la evaluación ergonómica el puesto de trabajo del alimentador de grano de sorgo mediante la guía técnica española, método OWAS y método RULA. La metodología empleada correspondió a una investigación de tipo exploratoria y descriptiva para detectar posibles riesgos a los que el trabajador estaba expuesto por la manipulación de cargas.

El nivel de riesgo obtenido de la evaluación, pronosticaba posible daño al sistema musculo-esquelético y se requería aplicar acciones correctivas inmediatamente, puesto que la carga aplicada en estas posturas tiene efectos dañinos sobre el trabajador.

Los resultados obtenidos fueron diversos: Con relación a la Guía Técnica Española fue el hallazgo de una ecuación matemática que permite predecir el nivel de riesgo de acuerdo a la magnitud de la carga. También se aplicó el software Autocad para registrar los ángulos generados en cada postura que se usan para el método RULA y se observó que los ángulos del software y los obtenidos de la medición directa tuvieron un ligero desfasamiento. Para reducir el riesgo del alimentador de sorgo al molino de martillos, se adaptó un dispositivo de transporte de grano del vehículo (camioneta) al molino de martillos que disminuye notablemente las posturas críticas del trabajador, así como el diseño y construcción de un dispositivo de tracto-carga.

Palabras clave: Ergonomía, Evaluación, Trabajador y Molienda de Sorgo

\begin{abstract}
This research presents an ergonomic study related to the need to modify the tasks traditionally performed in sorghum milling, taking as a basis the conservation of the health of the day laborer in his workplace, to propose an adaptation of the working conditions of operation, with the innovation of good practices in the production process. Initially, a description was made of the sorghum flour production process, which is used in the formulation of balanced feed for cattle, and then the ergonomic evaluation of the sorghum grain feeder's workplace was carried out using the Spanish technical guide, the OWAS method and the RULA method. The methodology used corresponded to an exploratory and descriptive type of research to detect possible risks to which the worker was exposed due to the handling of loads.

The level of risk obtained from the evaluation predicted possible damage to the musculoskeletal system and required immediate corrective actions, since the load applied in these postures has harmful effects on
\end{abstract}


the worker.

The results obtained were diverse: In relation to the Spanish Technical Guide was the finding of a mathematical equation that allows predicting the level of risk according to the magnitude of the load. Autocad software was also applied to record the angles generated in each posture used for the RULA method and it was observed that the angles of the software and those obtained from the direct measurement had a slight offset. To reduce the risk from the sorghum feeder to the hammer mill, a grain transport device was adapted from the vehicle (pickup truck) to the hammer mill that notably decreases the critical postures of the worker, as well as the design and construction of a tracto-loading device.

Key words: Ergonomics, Evaluation, Worker and Sorghum Milling.

\section{INTRODUCCIÓN:}

Según Díaz (2002) “Todo desarrollo productivo que involucra al hombre-máquina y el ambiente de trabajo, implican riesgos ocupacionales, los cuales pueden causar un daño físico al trabajador o a la propiedad", de lo anterior podemos entender como un daño físico a las lesiones, enfermedades ocupacionales, consecuencias mentales y nerviosas del organismo; que son contraídos en el medio ambiente laboral.

Según Mondelo, Torado y Bombard (2009) la evaluación de riesgos es un análisis sistemático de las condiciones y el ambiente de trabajo, que tiene por objeto identificar los posibles riesgos a los que el trabajador se expone en su área de trabajo ya sea dentro de las empresas o cualquier actividad productiva. Considerando lo antes expuesto, la productora de harina autorizó realizar la presente investigación mediante la evaluación ergonómica del alimentador de grano en el desempeño de sus actividades y de ser necesario proponer alguna alternativa de solución para el control de los riesgos derivados.

\section{TEORÍA:}

Con las metodologías escogidas se realizó la comparación de los resultados obtenidos para cuantificar la exposición del trabajador a movimientos repetitivos. Estos métodos son los siguientes: OWAS, RULA y Guía Técnica Española.

Osmos Karhu y Björn Trappe, desarrollaron un método para evaluar las posturas durante el desarrollo de las actividades del trabajador, el método se denomina OWAS y su aplicación proporciona buenos resultados, tanto en la mejora de la comodidad de los puestos de trabajo, como en el aumento de la calidad de vida del trabajador y mejora de la producción, como consecuencia ésta última a traído cambios en la educación laboral del trabajador en cuanto a la reducción de incidentes y accidentes en el área de trabajo (Karhu et al. 1981). Según Diego-Mas (2011), el método RULA fue desarrollado por los doctores McAtamney y Corlett en 1993 en la Universidad de Nottingham y por Institute for Occupational 
Ergonomics, que por sus siglas traducidas al español significan evaluación rápida de los miembros superiores. Mientras que la Guía técnica es parte de la legislación Española en materia de riesgos laborales

De acuerdo con Olivares et all (2021), esto es un hallazgo importante dado que con una ecuación de la recta obtenida a partir de un modelo de regresión lineal, permite determinar el porcentaje de protección o el nivel de daño de un trabajador sometido a cargas sobre el aparato musculo esquelético, de los cuales se encontró un nivel de riesgo elevado para el trabajador en el desempeño de sus tareas.

\section{PARTE EXPERIMENTAL:}

Para la elaboración de la harina se tiene la materia prima se procede a colocarla a pie de un molino de martillos como lo muestra la figura 1, Una vez accionado el molino, un trabajador (operador del puesto No. 1 "Alimentador de grano") figura 2 se encarga de abastecer de materia prima a la tolva de entrada de la maquina, cuyos requerimientos son de una tonelada por hora y para lo que se tiene que estar vaciando costales de sorgo en grano de $40 \mathrm{Kg}$. de peso cada 2.5 minutos aproximadamente. 
Figura 1 Tractor.

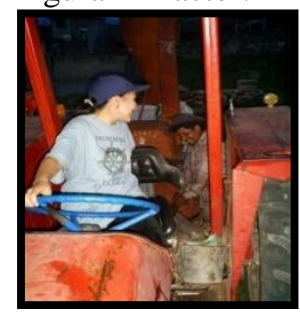

Figura 2: Molino

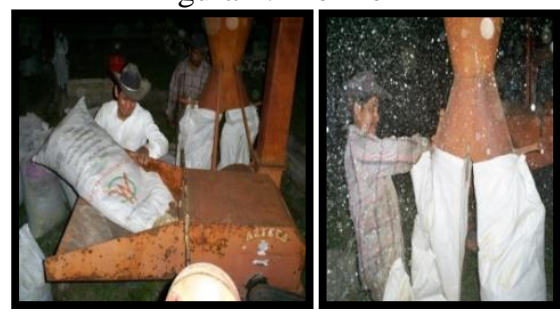

Figura 3: Envasador de harina.
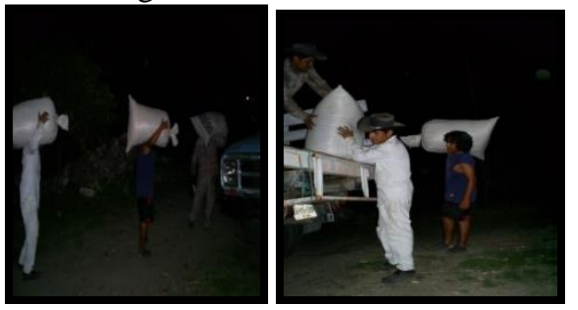

El molino desintegra el grano para la obtención de harina (ver Figura 3) y aspirada por un extractor que la dirige hasta un colector tipo ciclón. Otro trabajador (Operador del puesto No: 2 "Envasador de harina") Figura 3 recolecta la harina proveniente de las dos tolvas de descarga en costales de capacidad de $40 \mathrm{Kg}$. de peso, la frecuencia de la operación es la de estar cambiando los sacos cada 2.5 minutos colocando los costales llenos aproximadamente a 3 o 4 metros de distancia para su revisión y cierre del envase. Posteriormente se sellan los bultos llenos para que los estibadores (Operadores del puesto No. 3) carguen los bultos hacia el camión o la bodega (ver Figura 3), en un ciclo de aproximadamente 3 minutos por pieza (ver figura 3) Con los bultos de harina ya estibados se concluye la operación de molienda del sorgo.

Una vez conocido el proceso se procedió a efectuar la evaluación ergonómica de los trabajadores, desde la ubicación de la materia prima y su colocación sobre el molino de martillos. En la figura 4 se observa al trabajador sujetando un bulto de grano de sorgo de $40 \mathrm{~kg}$ a nivel de piso registrando los datos de las posturas:

Figura 4: Posiciones del alimentador de grano.
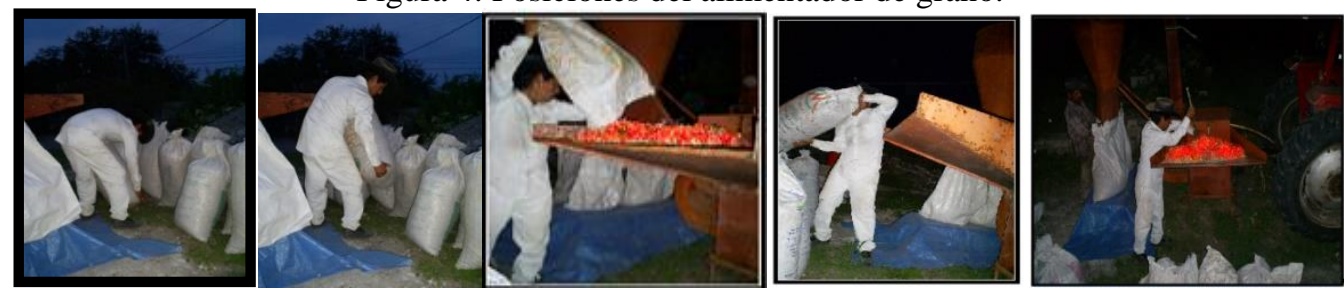

Posteriormente con el goniómetro se midió los ángulos de las diferentes posturas como son: el brazo, antebrazo, muñeca, giro de la muñeca, posición del cuello, como se encuentra posicionado el tronco con respecto a las piernas y a la carga que se está manejando figura 5. 


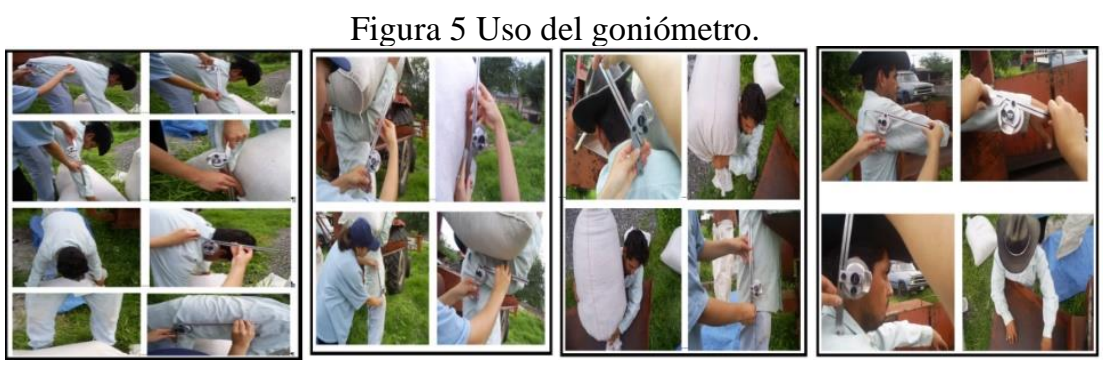

A continuación se presenta el registro de los datos posturales según la metodología empleada, pero por el espacio reducido en este artículo, solo mencionaremos los datos representativos sobre un trabajador mientras que el espacio muestral fue de 8 trabajadores:

Método OWAS

\begin{tabular}{|c|c|c|c|c|}
\hline & & $\begin{array}{l}\text { Prostoton } 1 \\
\text { Prazos }\end{array}$ & & \\
\hline Wo. De Trabaing & Espalda 4 & Erazos, & Plemens & Cargo \\
\hline 2 & 4 & 1 & 4 & 3 \\
\hline 3 & 4 & 1 & 4 & 3 \\
\hline 4 & 4 & 1 & 4 & 3 \\
\hline 5 & 4 & 1 & 4 & 3 \\
\hline 6 & 4 & 1 & 4 & 3 \\
\hline 7 & 4 & 1 & 4 & 3 \\
\hline s & 4 & 1 & 4 & 3 \\
\hline Promedio & 4 & 1 & 4 & 3 \\
\hline
\end{tabular}

Método Rula

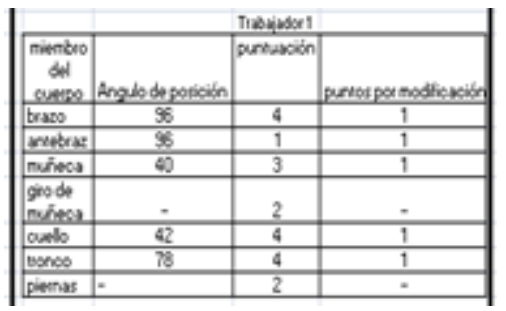

\section{Guía Técnica Española}

\begin{tabular}{|c|c|c|c|c|c|c|c|c|}
\hline & & & POSOCON1 & & & & & \\
\hline No. Thasibser & Prowed de skga (s) & 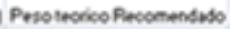 & Denplatumano versed & Geso del Thoenoso & Tpo de byert & 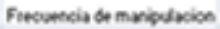 & Pesotold Sxio & Divencia of unupont. \\
\hline , & 1 is & $x$ & 0.87 & 09 & 1 & ois & $5 \quad 2000$ & \\
\hline 2 & at & 14 & 0.97 & 09 & 1 & o.s & $x \leqslant 0$ & c) \\
\hline 3 & 40 & w & osn & 09 & 1 & ous & 2000 & c \\
\hline 4 & (5) & $\mathbf{u}$ & on? & 08 & 1 & ors & 2050 & 0 \\
\hline 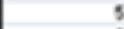 & 42 & w & $0: 84$ & 09 & 1 & 0.15 & 2000 & o \\
\hline 6 & 22 & w & 0.84 & 09 & i & 0.96 & 2000 & c \\
\hline ? & 4 & 14 & on & 09 & 1 & 0.15 & 2050 & \\
\hline : & 45 & n & on & 09 & $i$ & ous & 2000 & \\
\hline Phomesso & & w & on & 09 & 1 & ous & $x \leqslant 0$ & c \\
\hline
\end{tabular}

De acuerdo a la recolección de datos se obtuvo los siguientes resultados para las cinco posiciones iniciando con la Guía Técnica:

\begin{tabular}{|l|r|r|r|r|r|r|}
\hline \multicolumn{7}{|c|}{ Calculo de peso aceptable } \\
\hline Posicion & Peso Teorico $(\mathrm{Kg})$ & Desplazamiento vertical & Factor Giro & Factor Agarre & Factor Frecuencia & Peso Recomendado(Kg) \\
\hline 1 Recomendado & 14 & 1 & 0.9 & 1 & 0.85 & 10.71 \\
\hline 1 Maximo & 40 & 1 & 0.9 & 1 & 0.85 & 17.13 \\
\hline
\end{tabular}

Método OWAS: En este método se tuvieron los resultados observando la posición como lo muestra la figura 6 y la tabla de codificación asignando el valor correspondiente. 
Figura 6 Codificación para el método OWAS.

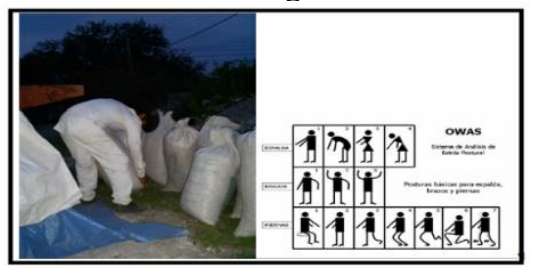

\begin{tabular}{c|c|c} 
Tabla Recolección de datos OWAS. \\
\hline Miembro del cuerpo & Posturas & Puntuación \\
Espalda & abajo-torcida & 4 \\
Brazos & bajo hombros & 1 \\
Piemas & flexion & 4 \\
Manejo de cargas & $>20 \mathrm{~kg}$ & 3 \\
\hline
\end{tabular}

Por lo tanto en la figura 7 se obtuvo la puntuación final de cuatro para el método OWAS.

Figura 7 Resultado final, posición uno para el método OWAS.

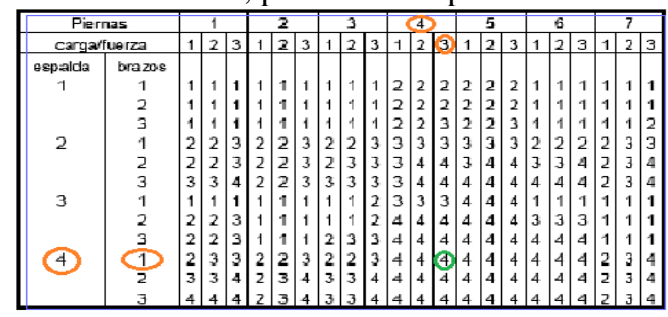

Método RULA: Para este método se obtuvieron todos los valores para los diferentes miembros del cuerpo (ver tabla A y B) obteniendo como puntuación final en la posición uno un valor de 7 como se aprecia en la figura 8.

\begin{tabular}{|c|c|c|c|}
\hline $\begin{array}{c}\text { Miembro del } \\
\text { cuerpo }\end{array}$ & \begin{tabular}{|c|}
$\begin{array}{c}\text { Angulo de } \\
\text { posición }\end{array}$ \\
\end{tabular} & Puntuación & $\begin{array}{l}\text { Puntos por } \\
\text { modificación }\end{array}$ \\
\hline Brazo & 96 & 4 & 1 \\
\hline Antebrazo & 96 & 1 & 1 \\
\hline $\begin{array}{l}\text { Murieca } \\
\text { Giro de muineca }\end{array}$ & 40 & 3 & 1 \\
\hline $\begin{array}{c}\text { Giro de munacas } \\
\text { Cvello }\end{array}$ & $\overline{41}$ & $\begin{array}{l}2 \\
4\end{array}$ & $i$ \\
\hline Tronco & 78 & 4 & 1 \\
\hline Piemas & & 2 & \\
\hline
\end{tabular}

Tabla B Resultados, RULA.

\begin{tabular}{c|c}
\hline Elemento de análisis & Puntuación \\
Miembros superiores & 7 \\
Miembros inferiores & 6 \\
Cargas y fuerzas & 3 \\
\hline
\end{tabular}

Figura 8 Resultado final, RULA.

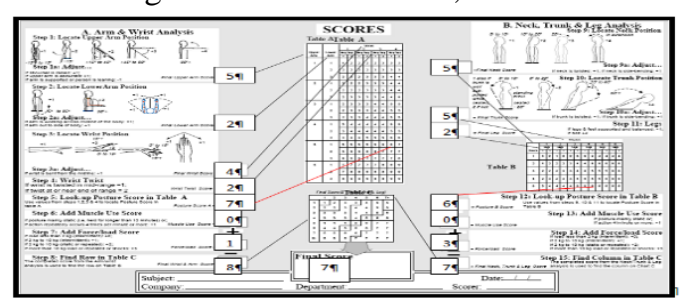


También se realizó un análisis con el programa AUTO-CAD y se analizaron las fotografías, acotando los ángulos, en especial la posición dos (miembros del cuerpo brazo, antebrazo y el tronco), observando lo siguiente: El ángulo calculado por AUTO-CAD mostrado en la figura 9, corresponde a la posición del brazo y fue de $27^{\circ}$ al compararlo con el de la tabla A se observa un diferencial

Figura 9 Estudio de la posición dos (brazo y ángulo) por AUTO CAD

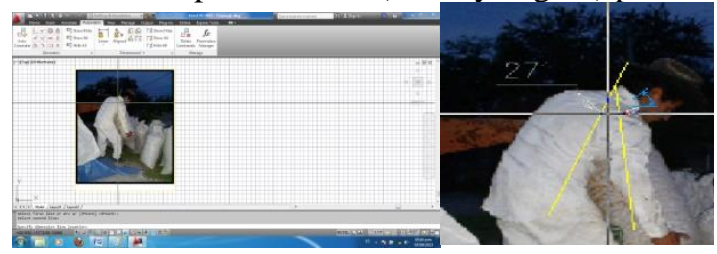

Con relación a la Guía Técnica Española se determino una ecuación matemática para predecir el porcentaje de la población protegida de acuerdo al manejo de la carga por el trabajador, así mismo se obtuvieron las gráficas para cada una de las posiciones adoptadas por el alimentador de sorgo. Al extrapolar los datos de la tabla de condiciones recomendadas para población protegida se pudo obtener un gráfico que representa una ecuación lineal de primer grado en la figura 10 de la forma y $=-\mathrm{X}+110$ la cual fue probada para las cinco posiciones con los datos obtenidos por la guía técnica española y que arrojo un comportamiento capaz de predecir la interacción entre el porcentaje de población protegida bajo el peso recomendado

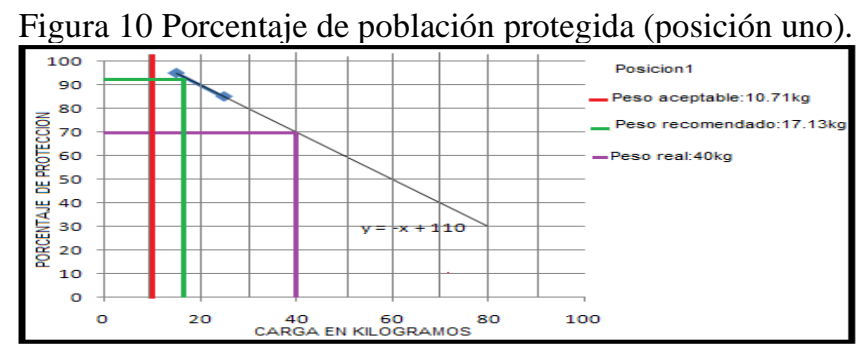

\section{CONCLUSIONES:}

Según el análisis realizado bajo la Guía Técnica española se obtienen valores de carga recomendados muy por debajo de la carga real aplicada a las diferentes posiciones del alimentador de grano. Por lo que se pueden generar lesiones músculo-tendinosas si el desarrollo de la actividad física se realiza en exceso. Se sugiere buscar alternativas para el desarrollo de la actividad. Mientras que el análisis realizado bajo el Método OWAS se obtuvo una puntuación final de 4 en los casos más críticos para las diferentes posiciones del alimentador de grano, que indica la metodología aplicar acciones correctivas inmediatamente, puesto que la carga causada por esta postura tiene efectos dañinos sobre el sistema músculo-esquelético. Se sugiere buscar alternativas para el desarrollo de la actividad. Con el análisis 
realizado bajo el Método RULA se obtienen una puntuación final de 7 que es la puntuación máxima establecida para las diferentes posiciones del alimentador de grano, que indica la metodología aplicar acciones correctivas inmediatamente, puesto que la carga causada por esta postura tiene efectos dañinos sobre el sistema músculo-esquelético. Se sugiere buscar alternativas para el desarrollo de la actividad. Un hallazgo más fue que los ángulos determinados bajo el Autocad tuvieron un breve desfasamiento con los registrados con ayuda de un goniómetro aplicándose a una de las posturas más representativas de la tarea, señalando de esta manera que es mucho más eficiente registrar los valores bajo la medición directa. Como se ve en la figura 11 que el trabajador realiza un menor esfuerzo, así como la postura adoptada no es tan crítica. Considerando que también se diseñó un dispositivo de tracto carga

Figura 11 Propuestas para reducción de riesgos de trabajadores agrícolas.

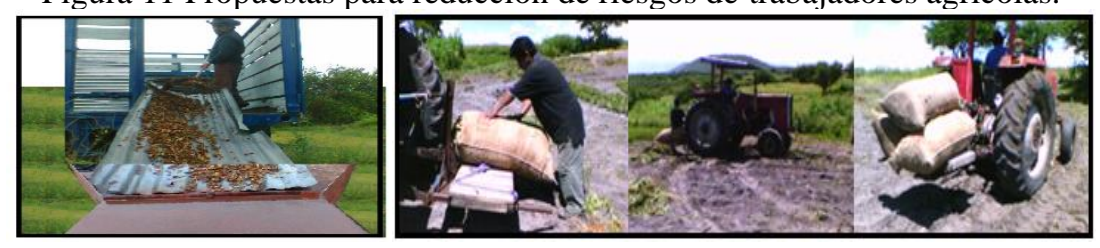




\section{BIBLIOGRAFÍA}

1. B. R. Juan Producción y expectativas del sorgo en México. D.F., México: SAGARPA. (2012).

2. Censo agropecuario en México (2007), INEGI.

3. Instituto Nacional de Seguridad e Higiene en el Trabajo INSHT (2003), Guía Técnica para la evaluación y prevención de los riesgos relativos a la manipulación manual de cargas. Barcelona, España: Ministerio del Trabajo.

4. M. C. Dasi, J. A. D. Mas, J. A. Marzal. Laboratorio de Ergonomía. Valencia, España: Alfaomega, (2011).

5. Olivares et all, (2021), "La ecuación de la recta en la ergonomía", South Florida Journal of Development, Miami, v.2, n.1, p.890-899-, jan./feb.2021. ISSN2675. 